Bull. Chem. Soc. Ethiop. 2017, 31(3), 535-544.

ISSN 1011-3924

(c) 2017 Chemical Society of Ethiopia and The Authors

Printed in Ethiopia

DOI: http://dx.doi.org/10.4314/bcse.v31i3.17

\title{
DABCO CONTAINING ACIDIC POLY(IONIC LIQUID): AN EFFICIENT CATALYST FOR THE ONE-POT PREPARATION OF 2,3-DIHYDROQUINAZOLINE-4(1H)-ONES
}

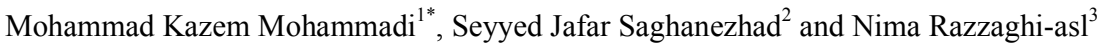 \\ ${ }^{1}$ Department of Chemistry, Ahvaz Branch, Islamic Azad University, Ahvaz, Iran \\ ${ }^{2}$ ACECR-Production Technology Research Institute, Ahvaz, Iran \\ ${ }^{3}$ Department of Medicinal Chemistry, School of Pharmacy, Ardabil University of Medical \\ Sciences, Ardabil, Iran
}

(Received November 24, 2017; Revised December 19, 2017; Accepted December 21, 2017)

\begin{abstract}
Diazabicyclo[2.2.2] octane (DABCO) containing acidic poly(ionic liquid) (DAIL) has been prepared via condensation of 1,4-dichloro butane and DABCO, as an efficient acidic catalyst and has been applied in the one-pot preparation of 2,3-dihydroquinazolin- $4(1 \mathrm{H})$-one derivatives. This catalyst has been characterized by FT-IR and TGA. According to the obtained results including time, yield and recyclability, DAIL could be considered as an efficient catalyst for organic transformations.
\end{abstract}

KEY WORDS: Poly(ionic liquid), Solvent-free, 2,3-Dihydroquinazolin-4(1H)-ones, DABCO, Multi-component reaction

\section{INTRODUCTION}

According to the simplicity, high yield of the products and one-pot synthesis, multi-component reactions (MCRs) have been greatly acknowledged. Due to the formation of multiple bonds in a continuous manner without the need for separation of intermediate adducts MCRs are gaining more attention lately [1-3]. Applications of homogeneous or heterogeneous catalysts or performing reaction in unconventional solvents are new strategies for obtaining desired products $[4,5]$.

Solvent-free organic reactions have been devised as an alternative tool for organic synthesis particularly from the viewpoint of green chemistry. Reduced pollution, lower costs, and simplicity in process and work-up are some advantages of solvent-free synthesis $[6,7]$.

Poly(ionic liquid)s (PILs) are considered as new materials with numerous potential applications in many fields such as energy and environment, analytical chemistry, materials science, biotechnology, catalysts or surface science $[8,9]$. The superiority of PILs over ionic liquids (ILs) are the enhanced mechanical stability, improved process ability, durability, and spatial controllability. Two basic strategies have been utilized for PILs synthesis, 1) direct polymerization of IL monomers and 2) post-modification of existing polymers [10]. Recently the scientists have focused on the preparation of nano-sized and nano-structured PILs [11]. Due to the diverse biological activities [12], synthesis of quinazolinone derivatives is a demanding task. Numerous synthetic methods have been developed for the synthesis of quinazolinone derivatives using silica sulfuric acid [13], gallium(III) triflate [12], MCM-41- $\mathrm{SO}_{3} \mathrm{H}$ [15], 1butyl-3-methylimidazolium tetrafluoroborate [16], molecular iodine [17], $\beta$-cyclodextrin [18], and metal-MWCNTs nanocomposites [19, 20]. Most of these methods suffer from one or more of these disadvantages, such as the use of hazardous organic solvents, low yields, strongly acidic conditions, expensive moisture-sensitive catalysts, and tedious work-up procedure [21]. Accordingly we decided to propose a greener procedure for the preparation of 2,3dihydroquinazolin- $4(1 \mathrm{H})$-one derivatives in the presence of catalytic amounts of DAIL under solvent-free conditions (Scheme 1).

*Corresponding author. E-mail: mohammadi@iauahvaz.ac.ir

This work is licensed under the Creative Commons Attribution 4.0 International License 


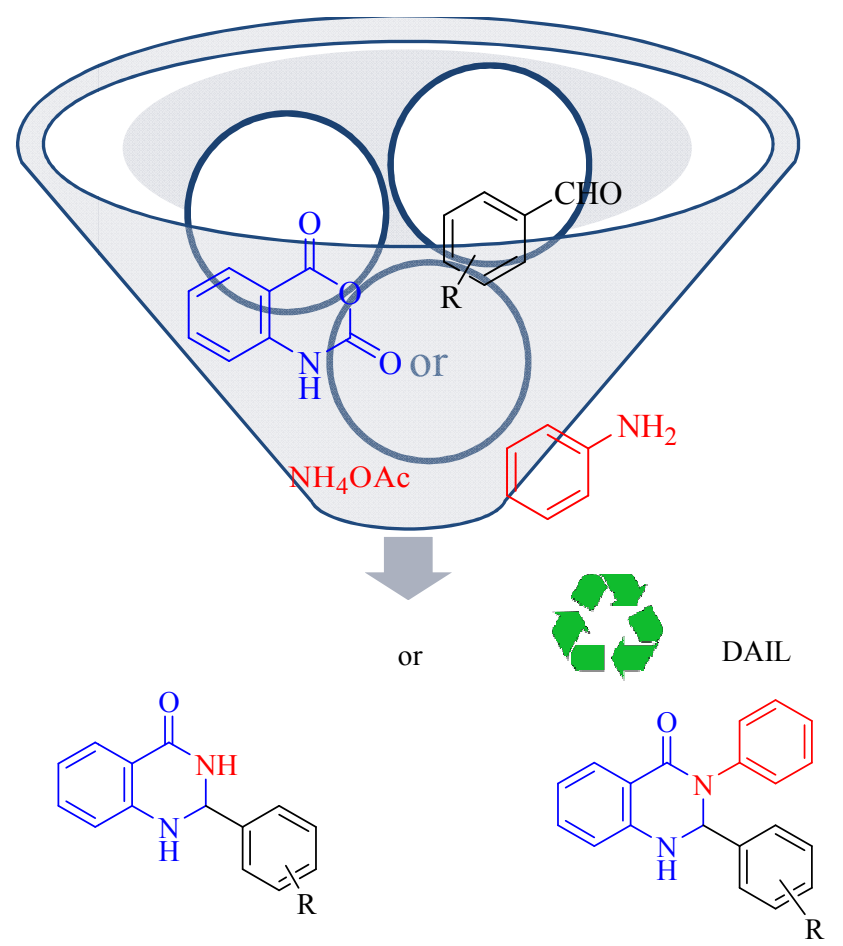

Scheme 1. Three-component reaction of isatoic anhydride, aromatic aldehydes and ammonium acetate/aniline for the one-pot preparation of 2-aryl-2,3-dihydroquinazolin- $4(1 \mathrm{H})$ ones and 2,3-disubstituted-2,3-dihydroquinazolin-4(1H)-ones.

\section{EXPRIMENTAL}

Materials and physical measurements

All commercially available chemicals were purchased from Fluka and Merck companies and used without further purification. Reaction monitoring was accomplished by TLC on silica gel polygram SILG/UV 254 plates. The IR spectra were recorded on a BOMEM MB-Series 1998 FT-IR spectrophotometer using $\mathrm{KBr}$ pellets for the samples and the catalyst in the range of $4000-400 \mathrm{~cm}^{-1}$. ${ }^{1} \mathrm{H}$ and ${ }^{13} \mathrm{C}$ NMR spectra were recorded in $\mathrm{CDCl}_{3}$ and DMSO- $d_{6}$ on a Bruker Advanced DPX $400 \mathrm{MHz}$ spectrometer using TMS as internal standard. The SEM analyses were carried out using a LEO 1455VP Scanning Electron Microscope, operating at 1-30 KV. CHNS$\mathrm{O}$ analysis was conducted by Costech elemental combustion system, ECS 4010.

General procedure for the preparation of DABCO containing acidic poly(ionic liquid) (DAIL)

In the first step, in a $50 \mathrm{~mL}$ three neck-round-bottom flask, DABCO $(1,4-$ diazabicyclo[2.2.2]octane) (10 mmol, $1.12 \mathrm{~g})$ was dissolved in ethylene glycol $(2.5 \mathrm{~mL})$ and under nitrogen atmosphere, 1,4-dichloro butane was added drop wise to the flask in $30 \mathrm{~min}$. Afterwards, the mixture was mixed for 8 hours at $110{ }^{\circ} \mathrm{C}$ to form the polymer. The obtained 
polymer was washed with dichloromethane and after drying in the oven at $60{ }^{\circ} \mathrm{C}$ for 12 hours, a white solid $(1.8 \mathrm{~g})$ was obtained.

The obtained solid was mixed with 2 equivalent of sulfuric acid drop wise in the dichloromethane. The mixture was refluxed for 48 hours under nitrogen atmosphere and the $\mathrm{HCl}$ gas was trapped in an alkaline solution. At last, DAIL was filtered and dried in the oven at $60^{\circ} \mathrm{C}$ for 12 hours.

\section{General procedure for the preparation of 2,3-dihydroquinazolin-4(1H)-one}

A mixture of isatoic anhydride $(1.0 \mathrm{mmol})$, aromatic aldehyde $(1.0 \mathrm{mmol})$, and ammonium acetate $(1.5 \mathrm{mmol})$ or aniline $(1.0 \mathrm{mmol})$, in the presence of DAIL $(0.01 \mathrm{~g})$ were thoroughly mixed and heated under solvent-free conditions at $90{ }^{\circ} \mathrm{C}$. Completion of the reaction was indicated by TLC [ethyl acetate/n-hexane (2:5)]. After completion of the reaction (as indicated in Table 2 and Table 3 ) the insoluble crude product was dissolved in hot ethanol and DAIL was removed by an external magnet. The filtrate was concentrated and the products were recrystallized from aqueous acetone or aqueous ethanol.

\section{RESULT AND DISSCUSSION}

In the first step, the catalyst was prepared by the nucleophilic attack of DABCO onto 1,4dichloro butane to form the adduct. To characterize the adduct the FT-IR spectrum was recorded. In the next step, the chloride anion was exchanged by $\mathrm{HSO}_{4}{ }^{-}$. To characterize the catalyst, the FT-IR spectrum was recorded. Accordingly the characteristic peak of $\mathrm{S}=\mathrm{O}$ between 1060 and $1228 \mathrm{~cm}^{-1}$ are evident. The thermo gravimetric analysis (TGA) of the catalyst was recorded too. The adsorbed water was released between $100-200{ }^{\circ} \mathrm{C}$. At about $200{ }^{\circ} \mathrm{C}$ the degradation of the catalyst backbone starts. The catalyst is completely decomposed at $500{ }^{\circ} \mathrm{C}$.

After Characterization of the catalyst, it was decided to evaluate its catalytic activity in the one-pot preparation of 2,3-dihydroquinazolin- $4(1 \mathrm{H})$-one derivatives. To optimize the reaction conditions, isatoic anhydride $(1.0 \mathrm{mmol})$, aromatic aldehyde $(1.0 \mathrm{mmol})$, ammonium acetate, and DAIL were mixed in a test tube under solvent-free conditions.

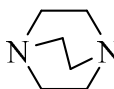<smiles>ClCCCCCl</smiles>

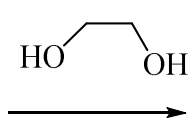

$110^{\circ} \mathrm{C}$
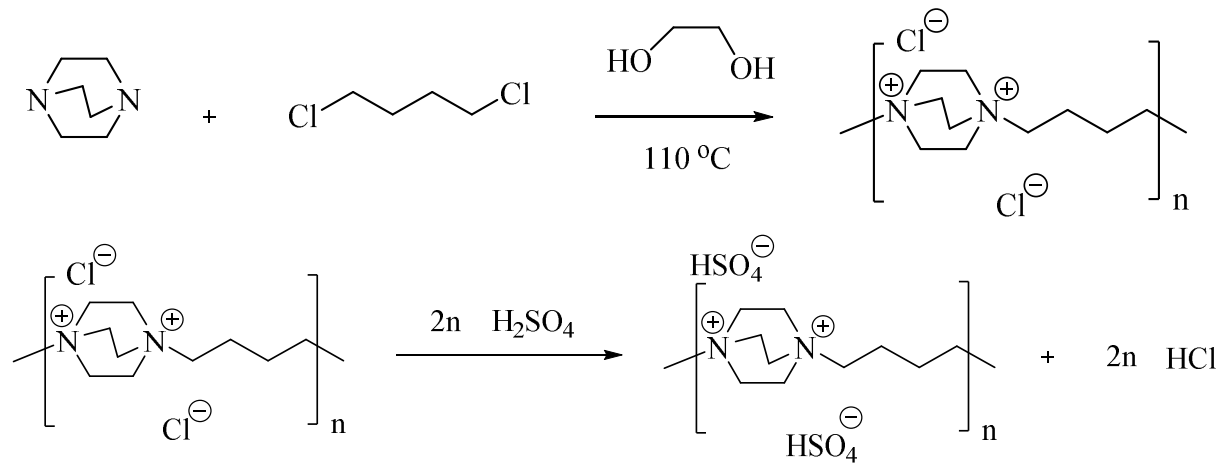

Scheme 2. Preparation of the DAIL catalyst. 


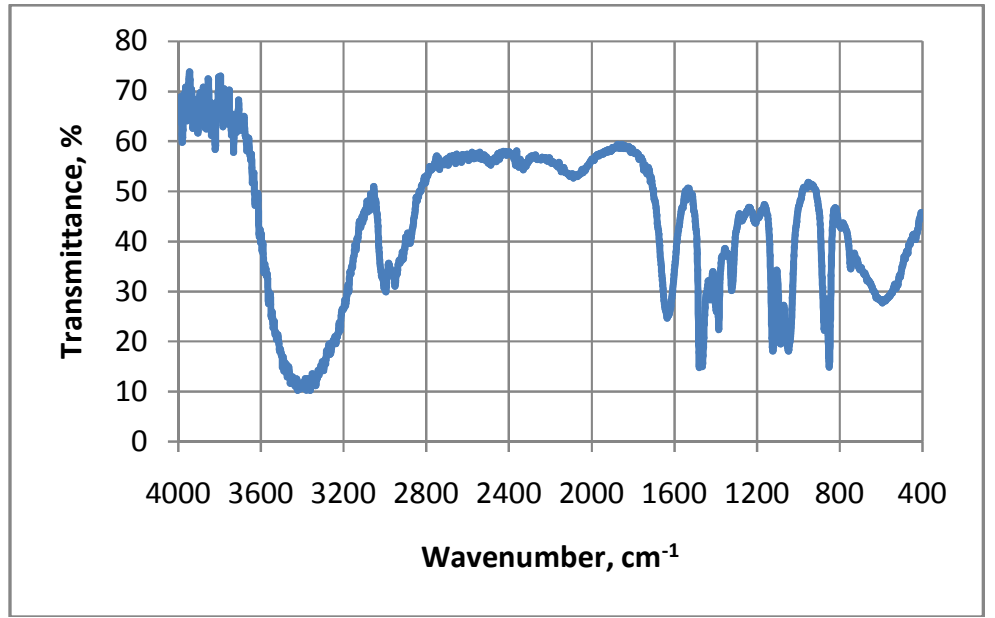

Figure 1. The FT-IR spectrum of the adduct of DABCO and 1,4-dichlorobutane.

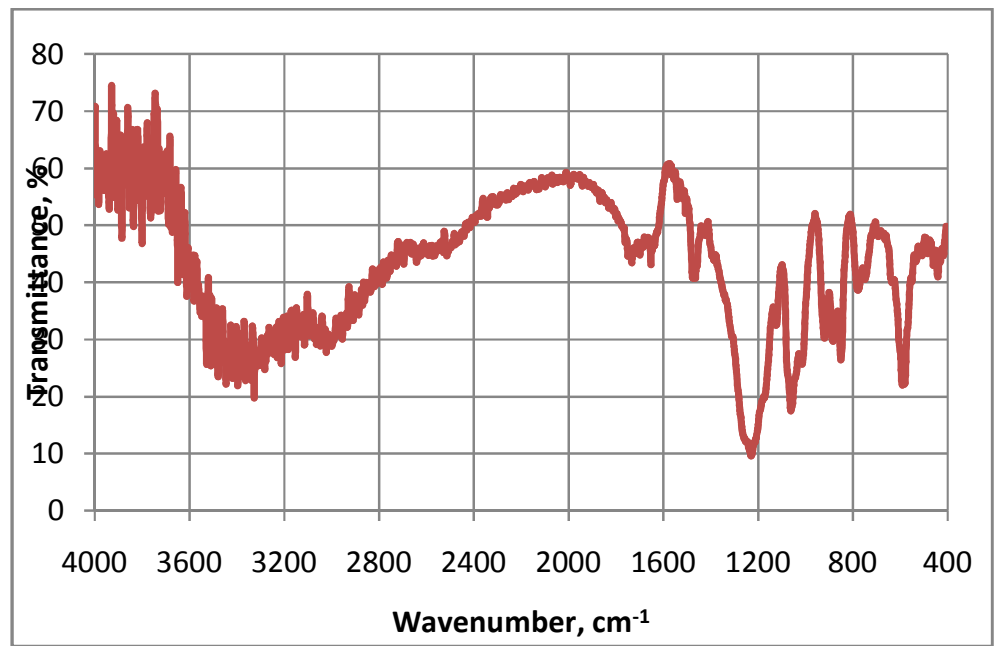

Figure 2. The FTIR spectrum of DAIL.

Table 1. Optimization of reaction conditions in the presence of DAIL for the reaction of isatoic anhydride $(1.0 \mathrm{mmol})$, aromatic aldehyde $(1.0 \mathrm{mmol})$ and ammonium acetate.

\begin{tabular}{|c|c|c|c|c|c|}
\hline Entry & Ammonium acetate (mmol) & Catalyst $(\mathrm{g})$ & $\mathrm{T}\left({ }^{\circ} \mathrm{C}\right)$ & Time $(\mathrm{min})$ & Yield $^{\mathrm{a}}(\%)$ \\
\hline 1 & 1.0 & - & 60 & 200 & - \\
\hline 2 & 1.0 & 0.01 & 60 & 45 & 15 \\
\hline 3 & 1.2 & 0.01 & 80 & 45 & 59 \\
\hline 4 & 1.5 & 0.01 & 90 & 30 & 95 \\
\hline 5 & 1.5 & 0.02 & 100 & 25 & 96 \\
\hline
\end{tabular}

${ }^{a}$ Yield corresponds to isolated yield.

Bull. Chem. Soc. Ethiop. 2017, 31(3) 


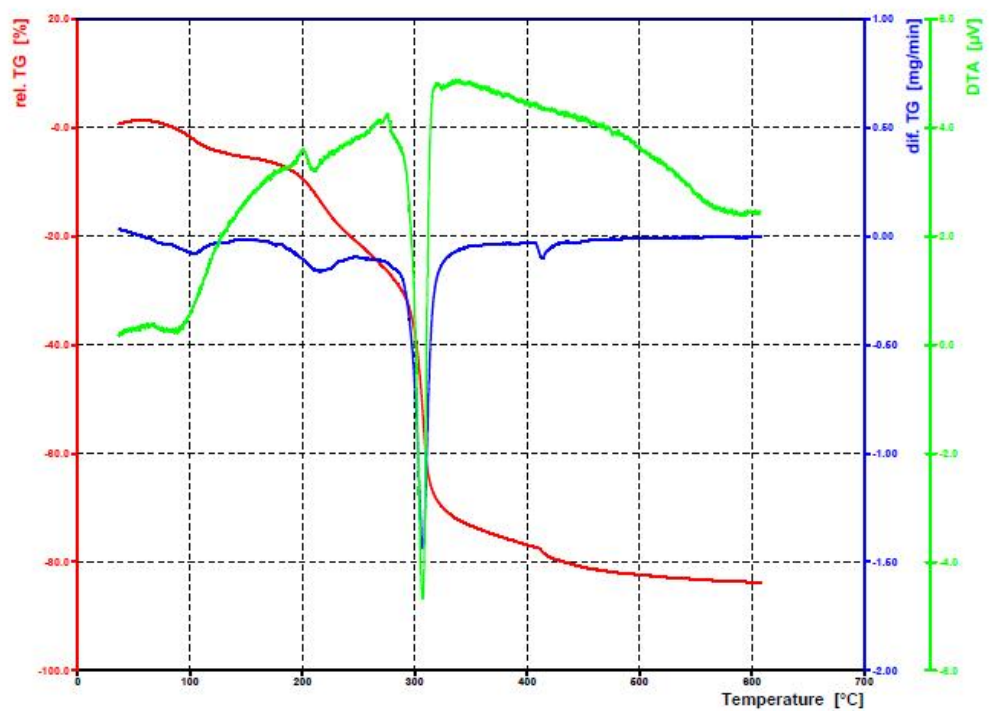

Figure 3. The TGA of DAIL.

The best results were obtained with the molar ratio of ammonium acetate $(1.5 \mathrm{mmol})$ and $0.01 \mathrm{~g}$ of the catalyst at $90^{\circ} \mathrm{C}$ under solvent-free conditions. With the optimum reaction conditions in hand, it was decided to evaluate the catalytic activity of DAIL, in the one-pot preparation of 2-aryl-2, 3-dihydroquinazolin-4(1H)-ones.

After the successful preparation of 2-aryl-2,3-dihydroquinazolin-4(1H)-ones, it was decided to broaden the scope of the catalyst by one pot preparation of 2,3-disubstituted-2,3dihydroquinazolin- $4(1 \mathrm{H})$-ones via the reaction of isatoic anhydride $(1.0 \mathrm{mmol})$,aromatic aldehyde $(1.0 \mathrm{mmol})$, aniline $(1 \mathrm{mmol})$, and DAIL $(0.01 \mathrm{~g})$ under solvent-free conditions. The results were summarized in Table 3.

It can be seen from Table 2 that with the presence of electron withdrawing groups in the aromatic aldehydes, time and the yield of products increased. This is because of electron withdrawing groups make better condition for the reaction of $\mathrm{NH}_{2}$ group with aldehydes.

The postulated mechanism has been drawn for the one-pot preparation of 2,3-disubstituted2,3-dihydroquinazolin-4(1H)-ones $(\mathbf{6 a - h})$. The reaction commences with the amidation of amine on isatoic anhydride with the release of carbon dioxide. Subsequently, imine formation followed by Michael addition leads to the corresponding products (Scheme 3).

With the increasing interest in green chemistry, the recyclability and reusability of the catalyst was studied in the preparation of 2-aryl-2,3-dihydroquinazolin-4(1H)-ones. After completion of the reaction of isatoic anhydride $(1.0 \mathrm{mmol})$, aromatic aldehyde $(1.0 \mathrm{mmol})$, ammonium acetate $(1.5 \mathrm{mmol})$; DAIL $(0.01 \mathrm{~g})$ was separated and washed with water, hot ethanol and dried. The catalyst was used for four subsequent cycles. Small decrease in the performance of the catalyst is observed in subsequent cycles (Figure 4). 
Table 2.One pot preparation of 2-aryl-2, 3-dihydroquinazolin-4(1H)-ones in the presence of DAIL

\begin{tabular}{|c|c|c|c|c|}
\hline Entry & Aldehyde & Product & Time (min) & Yield (\%) \\
\hline 1 & & & 30 & 95 \\
\hline 2 & & & 35 & 93 \\
\hline 3 & & & 35 & 92 \\
\hline 4 & & & 40 & 90 \\
\hline 5 & & & 45 & 88 \\
\hline 6 & & & 35 & 94 \\
\hline 7 & & & 45 & 87 \\
\hline
\end{tabular}

Bull. Chem. Soc. Ethiop. 2017, 31(3) 
Table 3. One pot preparation of 2,3-disubstituted-2,3-dihydroquinazolin-4(1H)-ones $(\mathbf{6 a}-\mathbf{h})$ in the presence of DAIL.

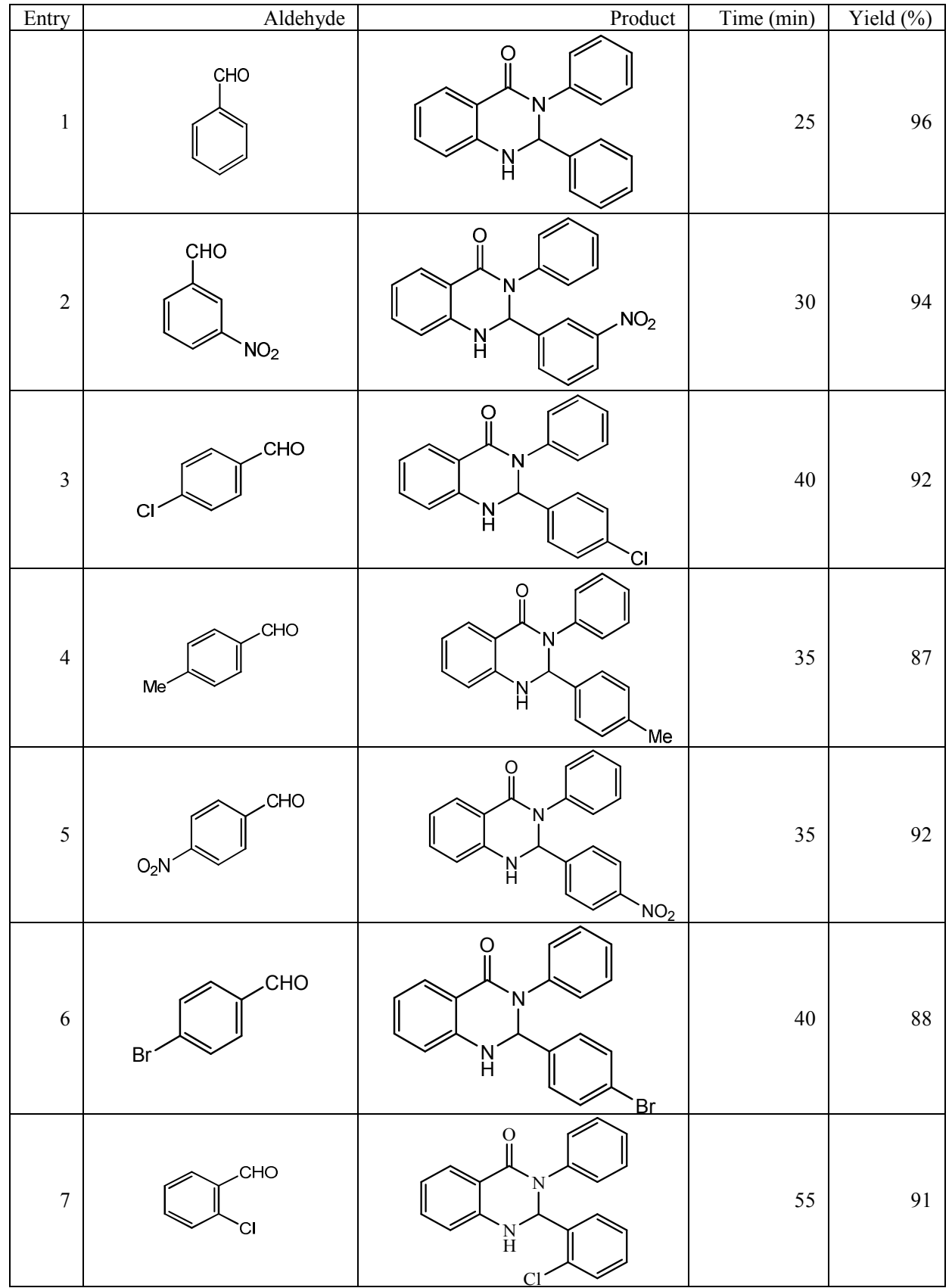

Bull. Chem. Soc. Ethiop. 2017, 31(3) 


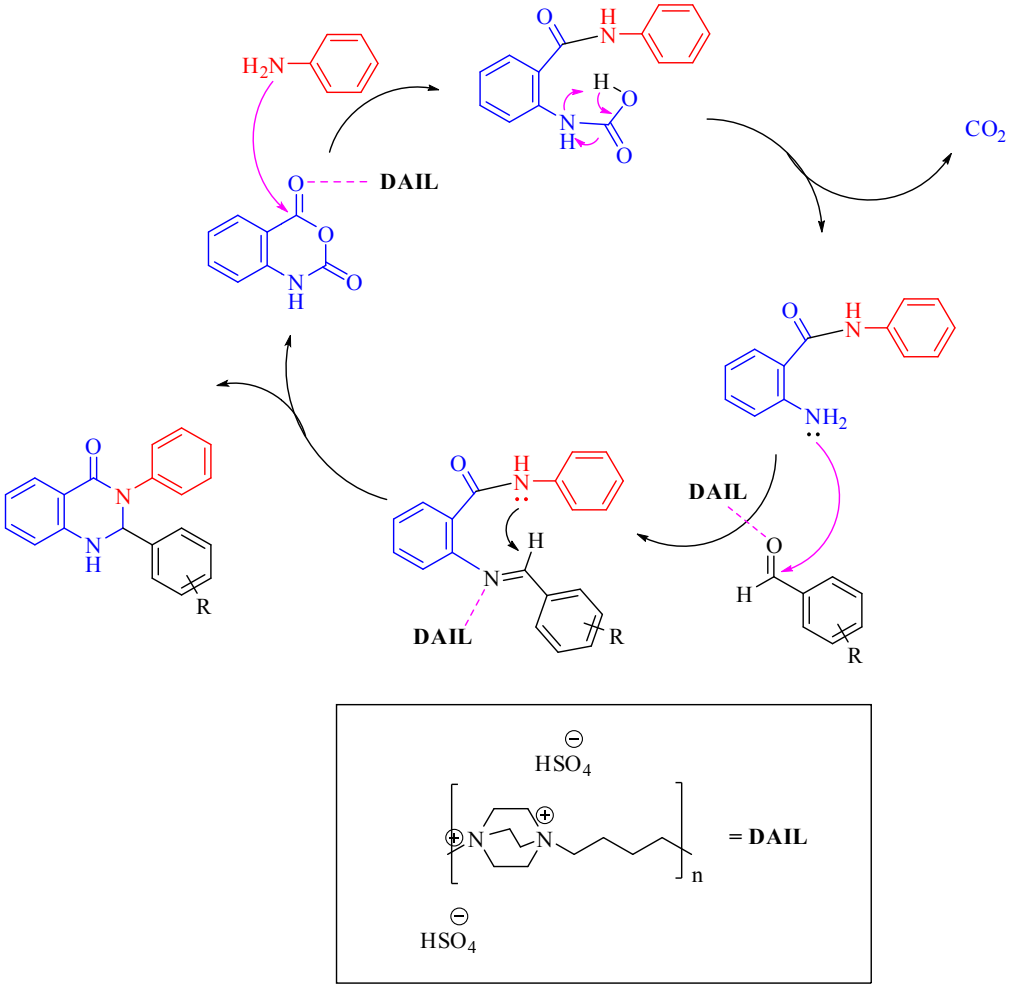

Scheme 3. Postulated mechanism.

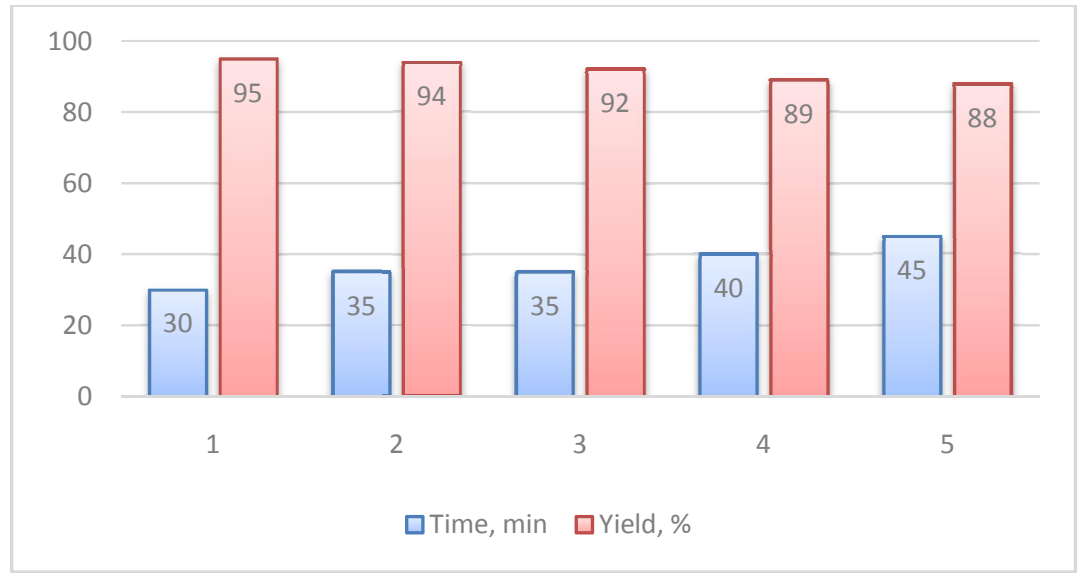

Figure 4. The recyclability of the catalyst in the reaction of isatoic anhydride $(1.0 \mathrm{mmol})$, aromatic aldehyde $(1.0 \mathrm{mmol})$, and ammonium acetate $(1.5 \mathrm{mmol})$ under solvent-free conditions.

Bull. Chem. Soc. Ethiop. 2017, 31(3) 


\section{CONCLUSION}

DABCO containing acidic poly(ionic liquid) (DAIL) has been utilized as an efficient acidic catalyst in the one-pot preparation of 2,3-dihydroquinazolin-4(1H)-one derivatives. According to the obtained results including time, yield and recyclability, DAIL could be considered as an efficient catalyst for organic transformations.

\section{ACKNOLEDGMENT}

The authors wish to thank the Ahvaz Branch, Islamic Azad University for the financial support.

\section{REFERENCES}

1. Dömling, A.; Ugi, I. Multicomponent reactions with isocyanides. Angew. Chem. Int. Ed. 2000, 39, 3168-3210.

2. Ganem, B. Strategies for innovation in multicomponent reaction. Design. Chem. Res. 2009, 42, 463-472.

3. Singh, M.S.; Chowdhury, S. Recent developments in solvent-free multicomponent reactions: A perfect synergy for eco-compatible organic synthesis. RSC Adv. 2012, 2, 4547-4592.

4. Climent, M.J.; Corma, A.; Iborra, S. Homogeneous and heterogeneous catalysts for multicomponent reactions. $R S C A d v$. 2012, 2, 16-58.

5. Gu,Y. Multicomponent reactions in unconventional solvents: State of the art. Green Chem. 2012, 14, 2091-2128.

6. Tanaka, K.; Toda, F. Solvent-free organic synthesis. Chem. Rev. 2000, 100, 1025-1074.

7. Alikarami, M.; Amozad, M. One-pot synthesis of 2,4,5-trisubstituted imidazole derivatives catalyzed by btppc under solvent-free conditions. Bull. Chem. Soc. Ethiop. 2017, 31, 177184.

8. Mecerreyes, D. Applications of ionic liquids in polymer science and technology. Prog. Polym. Sci. 2011, 36, 1629-1648.

9. Heravi, M.M.; Ansari, P.; Saeedi, M.; Tavakoli-Hosseini, N.; Narges Karimi, N. Green and practical synthesis of benzopyran and 3-sunstituted coumarin derivatives by Brønsted acid ionic liquid $\left[\left(\mathrm{CH}_{2}\right)_{4} \mathrm{SO}_{3} \mathrm{HMIM}\right]\left[\mathrm{HSO}_{4}\right]$. Bull. Chem. Soc. Ethiop. 2011, 25, 315-320.

10. Yuan, J. Antonietti M. Poly(ionic liquid)s: Polymers expanding classical property profiles. Polymer 2011, 52, 1469-1482.

11. Yuan, J.; Mecerreyes, D.; Antonietti, M. Poly(ionic liquid)s: An update. Progr. Polym. Sci. 2013, 38, 1009-1036.

12. Erlanson, D.A.; McDowell, R.S.; Brien, T.O. Fragment-based drug discovery. J. Med. Chem. 2004, 47, 3463-3482.

13. Dabiri, M.; Salehi, P.; Baghbanzadeh, M.; Zolfigol, M.A.; Agheb, M.; Heydari, S. Silica sulfuric acid: An efficient reusable heterogeneous catalyst for the synthesis of 2,3dihydroquinazolin- $4(1 \mathrm{H})$-ones in water and under solvent-free conditions. Catal. Commun. 2008, 9, 785-788.

14. Chen, J.X.; Wu, D.Z.; He, F.; Liu, M.C.; Wu, H.Y.; Ding, J.C.; Su, W.K. Gallium(III) triflate-catalyzed one-pot selective synthesis of 2,3-dihydroquinazolin-4(1H)-ones and quinazolin-4(3H)-ones. Tetrahedron Lett. 2008, 49, 3814-3822.

15. Rostamizadeh, S.; Amani, A.M.; Mahdavinia, G.H.; Sepehrian, H.; Ebrahimi, S. Synthesis of some novel 2-aryl substituted 2,3-dihydroquinazoline-4(1H)-ones under solvent-free condition via MCM-41- $\mathrm{SO}_{3} \mathrm{H}$ as a highly efficient sulfonic acid nanoreactor. Synthesis $\mathbf{2 0 1 0}$, $1356-1360$. 
16. Darvatkar, N.B.; Bhilare, S.V.; Deorukhkar, A.R.; Raut, D.G.; Salunkhe, M.M. [bmim] $\mathrm{HSO}_{4}$ : An efficient and reusable catalyst for one-pot three-component synthesis of 2,3-dihydro-4(1H)-quinazolinones. Green Chem. Lett. Rev. 2010, 3, 301-306.

17. Rostamizadeh, S.; Amani, A.M.; Aryan, R.; Ghaieni, H.R.; Shadjou, N. Synthesis of new 2aryl substituted 2,3-dihydroquinazoline-4(1H)-ones under solvent-free conditions, using molecular iodine as a mild and efficient catalyst. Synth. Commun. 2008, 38, 3567-3576.

18. Ramesh, K.; Karnakar, K.; Satish, G.; Reddy, K.H.V.; Nageswar, Y.V.D. N-Methylaniliniumtrifluoroacetate-promoted Prins reaction of $\alpha$-methylene- $\alpha$-tetralone dimers: Generation of new molecular scaffolds. Tetrahedron Lett. 2012, 53, 6095-6099.

19. Safari, J.; Gandomi-Ravandi, S. Application of the ultrasound in the mild synthesis of substituted 2,3-dihydroquinazolin-4(1H)-ones catalyzed by heterogeneous metal-MWCNTs nanocomposites. J. Mol. Struct. 2014, 1072, 173-178.

20. Nasseri, M.A.; Sadeghzadeh, .M. Diazabicyclo[2.2.2] octane stabilized on $\mathrm{Fe}_{3} \mathrm{O}_{4}$ as catalysts for synthesis of coumarin under solvent-free conditions. J. Iranian Chem. Soc. 2014, 11, $27-$ 33.

21. Kishore Kumar, V.; Kasi Viswanath, I.V.; Naga Raju, P.V.V.S.; Rajasekher, B.; Rama Dasu, B. Synthesis and in-vitro studies of some new quinoline 1,3,4-thiadiazolo pyrimidine derivatives. Bull. Chem. Soc. Ethiop. 2017, 31, 337-344. 